\title{
Association between thoracic fat measured using computed tomography and lung function in a population without respiratory diseases
}

\author{
Won Gun Kwack ${ }^{1,2}$, Yun-Seong Kang ${ }^{1}$, Yun Jeong Jeong ${ }^{1}$, Jin Young Oh ${ }^{1}$, Yoon Ki Cha ${ }^{3}$, Jeung Sook Kim , \\ Young Soon Yoon ${ }^{1}$
}

${ }^{1}$ Division of Pulmonary and Critical Care Medicine, Department of Internal Medicine, Dongguk University Ilsan Hospital, Goyang, South Korea; ${ }^{2}$ Division of Pulmonary, Allergy and Critical Care Medicine, Department of Internal Medicine, Kyung Hee University Hospital, Seoul, South Korea; ${ }^{3}$ Department of Radiology, Dongguk University Ilsan Hospital, Dongguk University, Goyang, South Korea

Contributions: (I) Conception and design: WG Kwack, YS Yoon; (II) Administrative support: None; (III) Provision of study materials or patients: None; (IV) Collection and assembly of data: WG Kwack, YS Kang; (V) Data analysis and interpretation: YJ Jeong, YK Cha; (VI) Manuscript writing: All authors; (VII) Final approval of manuscript: All authors.

Correspondence to: Young Soon Yoon, MD, PhD. Division of Pulmonary and Critical Care Medicine, Department of Internal Medicine, Dongguk University Ilsan Hospital, 27 Dongguk-ro, Ilsandong-gu, Goyang, Gyeonggido 10326, South Korea. Email: ysyoonmd@gmail.com.

Background: Local fat distribution patterns and their local or systemic effects have recently attracted significant attention. The aim of this study was to assess the impact of thoracic adiposity on lung function in a population without respiratory diseases according to sex.

Methods: A total of 455 subjects (282 males and 173 females), who had undergone spirometry, and chest and abdominal computed tomography between June 2012 and June 2016 at medical healthcare center, were included. Pericardial fat, intrathoracic fat, subcutaneous thoracic fat, and both visceral and subcutaneous abdominal fat were measured by directly assessing tissue volume using computed tomography. Multiple linear regression analyses adjusted for pack-years of smoking, high-density lipoprotein, and high-sensitivity C-reactive protein were performed to evaluate the association between fat volumes and lung function.

Results: In males, intrathoracic fat and visceral abdominal fat were inversely associated with forced expiratory volume in $1 \mathrm{~s}\left(\mathrm{FEV}_{1}\right) \%$ predicted $(\mathrm{P}=0.025, \mathrm{P}=0.010$, respectively), and subcutaneous thoracic fat volumes showed a negative correlation with both $\mathrm{FEV}_{1} \%$ and forced vital capacity (FVC) \% predicted $(\mathrm{P}=0.019, \mathrm{P}=0.045$, respectively). In females, subcutaneous thoracic fat demonstrated a negative correlation with both $\mathrm{FEV}_{1} \%$ and $\mathrm{FVC} \%$ predicted ( $\mathrm{P}=0.031$ and $\mathrm{P}=0.008$, respectively).

Conclusions: The influence of local thoracic fat distribution on lung function differed according to sex. Visceral fat and subcutaneous thoracic fat in males and subcutaneous fat in females were significantly associated with decreased lung function.

Keywords: Intrathoracic fat; subcutaneous fat; lung function

Submitted Aug 28, 2019. Accepted for publication Nov 12, 2019.

doi: $10.21037 /$ jtd.2019.11.54

View this article at: http://dx.doi.org/10.21037/jtd.2019.11.54

\section{Introduction}

Fat tissue, the so-called "new endocrine organ", has metabolic and mechanical effects on physical function (1-3). The accumulation of fat in specific parts of the body, including the upper body, and the central body or abdomen, has been shown to a crucial risk factor for cardiovascular and metabolic problems (4-6). Several previous studies have demonstrated that increased fat deposition around the heart is related to inflammatory marker levels and both morbidity and severity of cardiovascular diseases (7-10). 


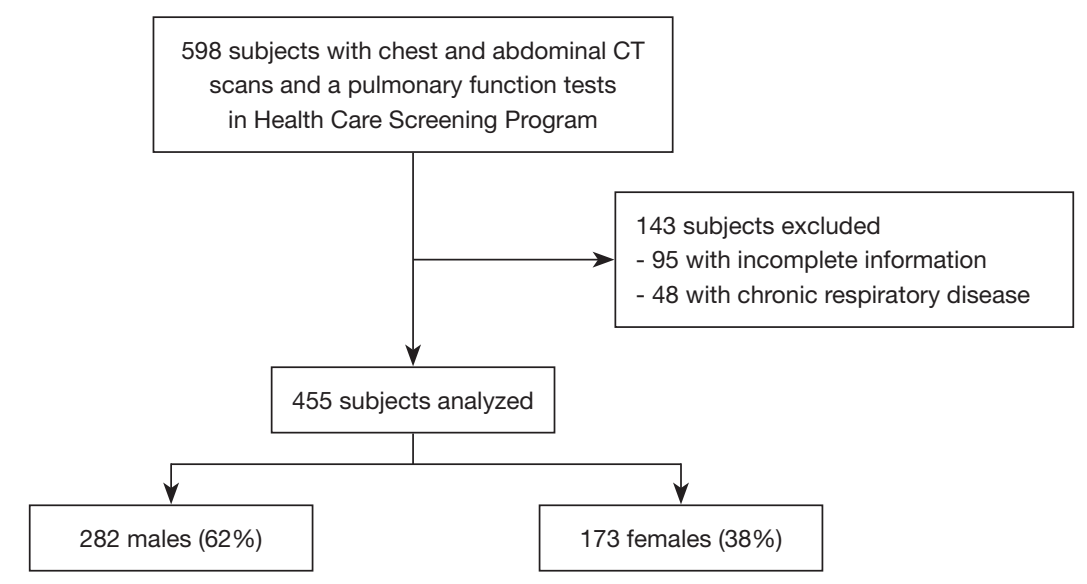

Figure 1 A flow diagram of the study population. CT, computed tomography.

An association between local fat disposition and lung function has been suggested. Increased thoracic fat volume assessed from the pericardial fat volume correlated with impaired lung function measured using pulmonary function test, particularly with a restrictive pattern (11). Given the close proximity of thoracic fat to the pulmonary vasculature and lung parenchyma, thoracic fat may affect lung function. Furthermore, markers of abdominal obesity, such as waist circumference and abdominal height, have been reported to be negatively associated with lung function in the general population (12). However, there were differences in the targeted local fat compartment and fat measurement methods in previous studies, which may have conflicting results regarding the relationship between lung function and thoracic fat, as well as abdominal fat $(13,14)$.

To the best of our knowledge, no study has examined the relationship between lung function and all types of thoracic visceral and subcutaneous fat, as well as abdominal fat in a single investigation. The aims of the present study, therefore, were to determine the association between lung function and all types of thoracic fat volumes as well as abdominal fat volumes assessed using computed tomography (CT) and to estimate differences according to sex.

\section{Methods}

\section{Study design and subjects}

This cross-sectional study included subjects between 18 and 80 years of age who underwent both chest and abdominal CT and a pulmonary function test within 2 days of a healthscreening program examination at the Medical Healthcare
Center of Dongguk University in South Korea from June 2012 to June 2016. All subjects voluntarily received the health screening to assess their health. Subjects with a history of chronic respiratory diseases (chronic obstructive pulmonary disease, asthma, interstitial lung disease, and primary or secondary lung malignancy) or those with incomplete information were excluded (Figure 1). The study protocol was approved by the Institutional Review Board of Dongguk University Ilsan Hospital (2016-146).

\section{Data collection}

Baseline characteristics, including the history of smoking and alcohol consumption, and underlying diseases (assessed by self-administered medical questionnaire), blood pressure, anthropometric values, including height, weight and waist circumference, body mass index (BMI), percentage body fat and blood test results, were obtained. Waist circumference was measured at the midpoint between the lower border of the rib cage and iliac crest, according to the World Health Organization recommendation (15). Body fat percentage was measured using a body composition analyzer (Inbody 720, Biospace, Seoul, Korea).

\section{Local fat volume measurements}

CT was performed with subjects in the supine position using a 64-channel multi-detector CT scanner (Siemens Medical Solutions, Forchheim, Germany). Fat volumes were measured using a dedicated offline workstation equipped with semi-automated image segmentation software implemented in the analysis software version 

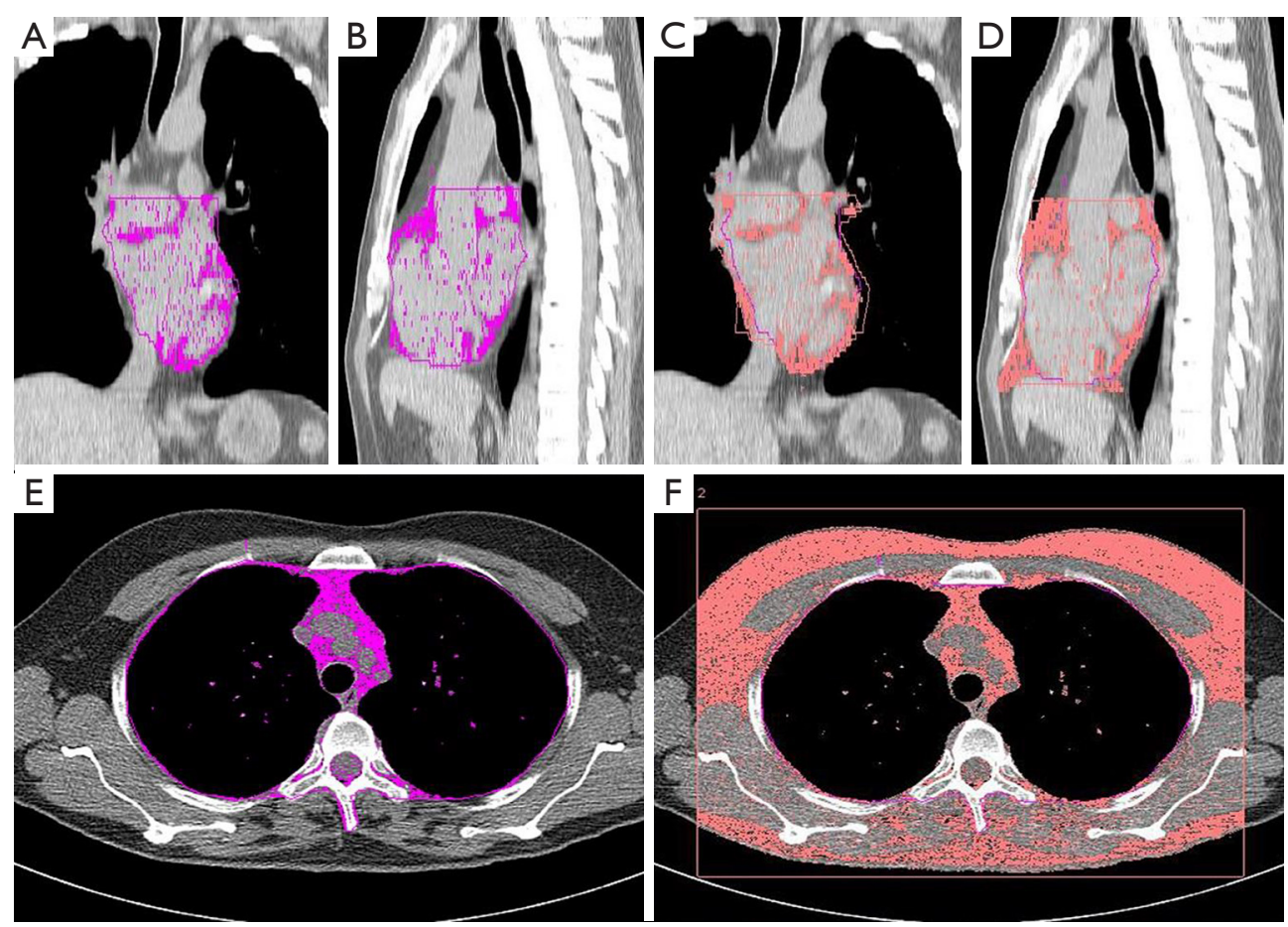

Figure 2 Trunk fat quantification using computed tomography. Semi-automated image segmentation was used for pericardial fat volume (A and B), total thoracic fat volume (C and D). Intrathoracic fat was calculated by subtracting pericardial fat from total thoracic fat. Thoracic subcutaneous adipose tissue was calculated by subtracting fat in the thoracic cage (E) from total adipose tissue at the levels of the $\operatorname{aortic} \operatorname{arch}(\mathrm{F})$.

10.0 (Biomedical Imaging Resource, Mayo Foundation, Rochester, MN, USA). Thoracic fat volumes included pericardial fat, intrathoracic fat, and subcutaneous thoracic fat. Abdominal fat volumes included visceral and subcutaneous abdominal fat. Intrathoracic fat and pericardial fat, which are considered as the thoracic visceral fat, were defined as previously described $(9,16)$. Briefly, total thoracic fat refers to any fat tissue located within the thorax from the level of the bifurcation of the pulmonary artery trunk to the diaphragm, and from the anterior chest wall to the descending aorta. Pericardial fat refers to all fat tissue located within the pericardial sac, with the same cranial and caudal boundaries. Intrathoracic fat volume was assessed by subtracting pericardial fat volume from total thoracic fat volume (Figure $2 A, B, C, D)(9,17)$. Although there is little consensus about how to measure subcutaneous thoracic fat, we measured it on the first axial CT image above the aortic arch. First, the superior aspect of the aortic arch was visually identified, and then the first axial image above the arch was selected by scrolling toward the apex of the lungs. Its volume was calculated by subtracting the fat tissue volume in the thoracic cavity from the entire fat tissue volume in the predefined lateral boundary in the first axial image above the arch, as previously described (Figure 2E,F) (18). With manual segmentation, the lateral boundary was outlined at the lateral edge of the scapular bone because a considerable number of object slices could not cover the entire circumference of the chest; in addition, it enabled the inclusion of the maximum possible area Pixels with Hounsfield unit (HU) values of -200 to -40 within the selected region were defined as adipose tissue in the thorax (19). For abdominal adipose tissue, volume analysis was performed on axial CT images at the level of the umbilicus, with a threshold of -250 and $-50 \mathrm{HU}$. Visceral abdominal fat was defined as any intra-abdominal adipose tissue confined to within the parietal peritoneum or the transversalis fascia. Subcutaneous abdominal fat volume was assessed by subtracting the visceral abdominal fat volume from the entire fat volume at that level $(16,20)$. All fat volumes are reported in $\mathrm{cm}^{3}$. To evaluate inter-reader reproducibility, thoracic and abdominal fat volumes were repeatedly measured in 48 randomly selected subjects by 
Table 1 Baseline characteristics of the participants according to sex

\begin{tabular}{lccc}
\hline Variable & Male $(\mathrm{n}=282)$ & Female $(\mathrm{n}=173)$ & $\mathrm{P}$ value \\
\hline Median age [IQR], years & $54[42-67]$ & $55[43-67]$ & 0.398 \\
Systolic blood pressure, mmHg & $123.42 \pm 13.41$ & $121.28 \pm 14.06$ & 0.105 \\
Hypertension & $56(19.9)$ & $21(12.1)$ & 0.033 \\
Diabetes mellitus & $38(13.5)$ & $10(5.8)$ & 0.009 \\
Current and ex-smoker & $144(51.1)$ & $38(22.0)$ & $<0.001$ \\
Pack-years of smoking (n=182) & $11.18 \pm 14.65$ & $3.95 \pm 8.71$ & $<0.001$ \\
Heavy drinker & $13(7.2)$ & $6(5.4)$ & 0.530 \\
hsCRP, mg/dL & $0.20 \pm 0.42$ & $1.08 \pm 11.47$ & 0.213 \\
HDL, mg/dL & $51.62 \pm 15.62$ & $57.34 \pm 17.00$ & $<0.001$ \\
LDL, mg/dL & $134.0 \pm 2.24$ & $130.0 \pm 2.70$ & 0.261 \\
HbA1C, \% & $5.84 \pm 0.06$ & $5.69 \pm 0.04$ & 0.579 \\
ILD pattern on chest CT & $4(1.4)$ & $0(0)$ & 0.303 \\
\hline
\end{tabular}

Data presented as mean $\pm \mathrm{SD}$, or $\mathrm{n}(\%)$ unless otherwise indicated. IQR, interquartile range; hsCRP, high sensitivity C-reactive protein; HDL, high-density lipoprotein; LDL, low-density lipoprotein; HbA1C, glycated hemoglobin; ILD, interstitial lung disease; CT, computed tomography. "Defined as 3 bottles of Soju/week.

two independent readers. The inter-reader concordance correlation coefficients for pericardial, intrathoracic, visceral abdominal, and subcutaneous abdominal fat volumes were $0.91,0.87,0.77$, and 0.78 respectively.

\section{Pulmonary function test}

Spirometry was performed by experienced technicians using a spirometer (Vmax 2130, Sensormedics, Yorba Linda, CA, USA) in the standing position, in accordance with American Thoracic Society recommendations (21). Forced vital capacity (FVC) and forced expiratory volume in $1 \mathrm{~s}\left(\mathrm{FEV}_{1}\right)$ were assessed in absolute values $(\mathrm{L})$ and the $\mathrm{FEV}_{1} / \mathrm{FVC}$ ratio was measured in percentages. Predicted spirometry values (\%) were calculated using a formula based on the Korean population (22).

\section{Statistical analyses}

All continuous and categorical variables were compared using the Student's $t$-test or Mann-Whitney U test, and the chi-squared test, respectively. Associations among thoracic fat volumes, abdominal fat volumes, and traditional obesity indices were assessed using Pearson's correlation analysis. The associations between lung functions and fat volumes were examined using multiple linear regression analyses as well as the associations between lung functions and logtransformed fat volumes in subgroup analyses according to sex. Pack-years of smoking, high-density lipoprotein (HDL), and high sensitivity C-reactive protein (hsCRP) were adjusted for $\mathrm{FEV}_{1} \%$ predicted and $\mathrm{FVC} \%$ predicted. Also, age, height, pack-years of smoking, HDL, and hsCRP were used as adjusted variables for the $\mathrm{FEV}_{1} / \mathrm{FVC}$ ratio. For estimating the heterogeneity by sex, interaction effects in a generalized linear model were used. The inter-reader reproducibility was evaluated using intra-class correlation coefficients; $\mathrm{P}<0.05$ was statistically significant. All statistical analyses were performed using SPSS version 23.0 for Windows (IBM Corporation, Armonk, NY, USA).

\section{Results}

\section{Subject characteristics}

In total, 282 males (62\%) and 173 females participated in the present study. The subjects' demographic information and clinical characteristics according to sex are summarized in Table 1. There were more current and former smokers among males than among the females. Males had a significantly higher BMI and larger waist circumferences than females, although the percentage of body fat was 
Table 2 Anthropometry and lung function according to sex

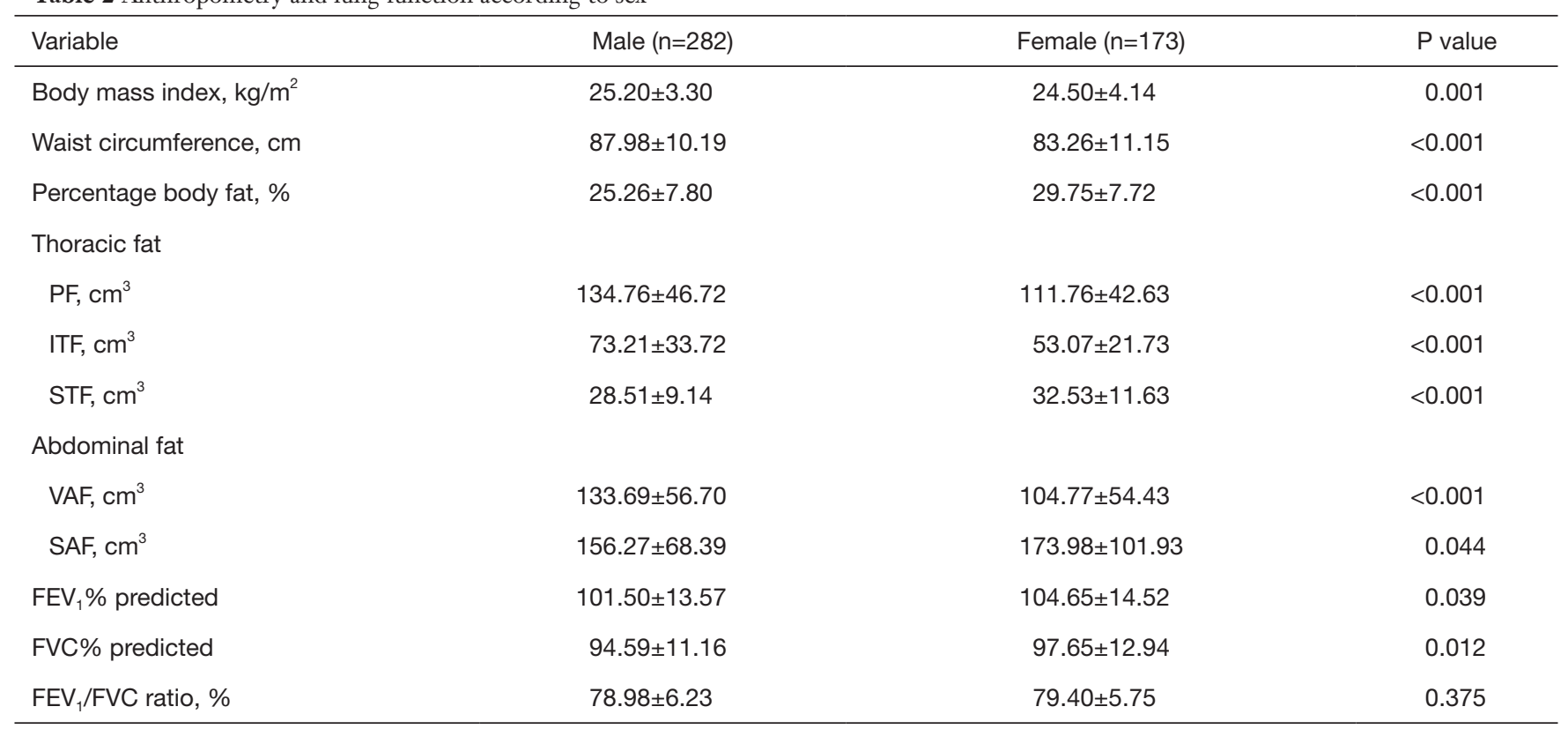

Data presented as mean \pm SD unless otherwise indicated. PF, pericardial fat; ITF, intrathoracic fat; STF, subcutaneous thoracic fat; VAF, visceral abdominal fat; SAF, subcutaneous abdominal fat; FVC, forced vital capacity; FEV $_{1}$, forced expiratory volume in $1 \mathrm{~s}$.

Table 3 Correlation coefficients between anthropometric indicators according to sex

\begin{tabular}{lcccccccc}
\hline Indicator & PF & ITF & STF & VAF & SAF & BMI & WC & Body fat percentage \\
\hline PF & 1 & 0.688 & 0.565 & 0.605 & 0.429 & 0.606 & 0.616 & 0.530 \\
ITF & 0.575 & 1 & 0.464 & 0.560 & 0.334 & 0.520 & 0.546 & 0.397 \\
STF & 0.481 & 0.312 & 1 & 0.373 & 0.762 & 0.748 & 0.635 & 0.813 \\
VAF & 0.676 & 0.604 & 0.496 & 1 & $0.138^{*}$ & 0.450 & 0.569 & 0.327 \\
SAF & 0.382 & 0.260 & 0.767 & 0.370 & 1 & 0.775 & 0.650 & 0.717 \\
BMI & 0.585 & 0.407 & 0.757 & 0.596 & 0.735 & 1 & 0.882 & 0.645 \\
WC & 0.563 & 0.423 & 0.612 & 0.584 & 0.578 & 0.747 & 1 & 0.522 \\
Body fat percentage & 0.283 & 0.193 & 0.621 & 0.412 & 0.570 & 0.499 & 0.386 & 1 \\
\hline
\end{tabular}

The upper right part refers to females, and the lower left part refers to males. ${ }^{*} \mathrm{P}=0.071$, otherwise $\mathrm{P}<0.05$. $\mathrm{PF}$, pericardial fat; ITF, intra thoracic fat; STF, subcutaneous thoracic fat; VAF, visceral abdominal fat; SAF, subcutaneous abdominal fat; BMI, body mass index; WC, waist circumference.

significantly higher in females than in males (Table 2). There was significantly higher visceral thoracic fat (pericardial fat and intrathoracic fat) and visceral abdominal fat volumes in males than in females. In contrast, there were significantly higher subcutaneous fat volumes in females rather than males in both the thorax and abdomen. Lung function, represented by $\mathrm{FEV}_{1} \%$ predicted and $\mathrm{FVC} \%$ predicted, was higher in females than in males.

\section{Association between regional fat volumes and lung function}

All surrogate markers of adiposity demonstrated a statistically significant positive association with one another; however, visceral abdominal fat and subcutaneous abdominal fat volumes exhibited no correlation in females (Table 3). Multiple regression coefficients for the relationship between fat volumes and lung function $\left(\mathrm{FEV}_{1} \%\right.$ predicted, $\mathrm{FVC} \%$ predicted, and the $\mathrm{FEV}_{1} / \mathrm{FVC}$ ratio) 
Table 4 Association between indicators of local fat deposits and lung function

\begin{tabular}{lllr}
\hline Variable & FEV $_{1} \%$ predicted $^{*}$ & FVC $\%$ predicted $^{*}$ & FEV $_{1} / \mathrm{FVC}_{(\%)}{ }^{\dagger}$ \\
\hline PF $\left(\mathrm{cm}^{3}\right)$ & $-0.013 \pm 0.015(0.374)$ & $-0.029 \pm 0.013(0.020)$ & $0.007 \pm 0.006(0.249)$ \\
ITF $\left(\mathrm{cm}^{3}\right)$ & $-0.063 \pm 0.022(0.004)$ & $-0.070 \pm 0.019(0.001)$ & $-0.003 \pm 0.010(0.720)$ \\
STF $\left(\mathrm{cm}^{3}\right)$ & $-0.232 \pm 0.065(0.001)$ & $-0.192 \pm 0.056(0.001)$ & $-0.040 \pm 0.028(0.150)$ \\
VAF $\left(\mathrm{cm}^{3}\right)$ & $-0.020 \pm 0.013(0.123)$ & $-0.029 \pm 0.011(0.007)$ & $0.002 \pm 0.019(0.705)$ \\
SAF $\left(\mathrm{cm}^{3}\right)$ & $-0.026 \pm 0.008(0.001)$ & $-0.020 \pm 0.007(0.003)$ & $-0.006 \pm 0.003(0.057)$ \\
\hline
\end{tabular}

Each association between fat volumes and lung function were evaluated in a separate multiple regression analysis ( $\mathrm{N}=455)$. Data presented as regression coefficients \pm standard error (P value). *Adjusted for pack-years of smoking, HDL, and hsCRP. ${ }^{\dagger}$ Adjusted for age, height, pack-years of smoking, HDL, and hsCRP. HDL, high-density lipoprotein; hsCRP, high sensitivity C-reactive protein; PF, pericardial fat; ITF, intra-thoracic fat; TF, total thoracic fat; STF, subcutaneous thoracic fat; VAF, visceral abdominal fat; SAF, subcutaneous abdominal fat; $F V_{1}$, forced expiratory volume in $1 \mathrm{~s}$; FVC, forced vital capacity.

Table 5 Association between indicators of log-transformed local fat deposits and lung function

\begin{tabular}{|c|c|c|c|}
\hline Variable & $\mathrm{FEV}_{1} \%$ predicted $^{*}$ & FVC\% predicted* & $\mathrm{FEV}_{1} / \mathrm{FVC}(\%)^{\dagger}$ \\
\hline $\mathrm{PF}\left(\mathrm{cm}^{3}\right)$ & $-2.504 \pm 4.814(0.603)$ & $-5.093 \pm 3.991(0.203)$ & $0.646 \pm 2.234(0.773)$ \\
\hline $\operatorname{ITF}\left(\mathrm{cm}^{3}\right)$ & $-9.011 \pm 4.006(0.025)^{\ddagger}$ & $-8.882 \pm 3.317(0.008)$ & $-2.158 \pm 1.859(0.247)^{\ddagger}$ \\
\hline $\operatorname{STF}\left(\mathrm{cm}^{3}\right)$ & $-13.119 \pm 5.544(0.019)$ & $-9.303 \pm 4.620(0.045)$ & $-6.578 \pm 2.442(0.008)^{\ddagger}$ \\
\hline $\mathrm{SAF}\left(\mathrm{cm}^{3}\right)$ & $-6.520 \pm 4.100(0.113)$ & $-2.299 \pm 3.420(0.502)$ & $-6.678 \pm 1.765(0.001)^{\ddagger}$ \\
\hline \multicolumn{4}{|c|}{ Female $(n=173)$} \\
\hline $\mathrm{PF}\left(\mathrm{cm}^{3}\right)$ & $0.042 \pm 6.913(0.995)$ & $-3.134 \pm 6.064(0.606)$ & $0.048 \pm 2.635(0.985)$ \\
\hline ITF $\left(\mathrm{cm}^{3}\right)$ & $0.600 \pm 5.823(0.918)^{\ddagger}$ & $-3.409 \pm 5.106(0.505)$ & $1.550 \pm 2.208(0.483)^{\ddagger}$ \\
\hline
\end{tabular}

Each association between fat volumes and lung function were evaluated in a separate multiple regression analysis. Log-transformed fat volumes were used. Data presented as regression coefficients \pm standard error ( $\mathrm{P}$ value). *Adjusted for pack-years of smoking, HDL, and hsCRP. ${ }^{\dagger}$ Adjusted for age, height, pack-years of smoking, HDL, and hsCRP. ${ }^{\ddagger} \mathrm{P}$ value for the interaction of sex to the association $\left(\mathrm{P}_{\text {interaction }}\right.$ $<0.05)$. HDL, high-density lipoprotein; hsCRP, high sensitivity C-reactive protein; PF, pericardial fat; ITF, intra-thoracic fat; TF, total thoracic fat; STF, subcutaneous thoracic fat; VAF, visceral abdominal fat; SAF, subcutaneous abdominal fat; $F_{E V}$, forced expiratory volume in 1 s; FVC, forced vital capacity.

were summarized in Table 4. All thoracic fat and abdominal fat volumes showed a negative association with lung function (all $\mathrm{P}<0.05)$. Multiple regression coefficients for the relationship between log-transformed fat volumes and lung function according to sex were represented in Table 5 . In subgroup analyses according to sex, intrathoracic fat, subcutaneous thoracic fat volumes, and visceral abdominal fat were inversely associated with both $\mathrm{FEV}_{1} \%$ predicted and $\mathrm{FVC} \%$ predicted in males (all $\mathrm{P}<0.05)$. In females, subcutaneous thoracic fat volumes were inversely associated with both $\mathrm{FEV}_{1} \%$ predicted and $\mathrm{FVC} \%$ predicted $(\mathrm{P}=0.031$, and $\mathrm{P}=0.008$, respectively). Among these associations, the sex factor showed statistically significant interaction effects on the relationship between intrathoracic fat, visceral abdominal fat volumes and $\mathrm{FEV}_{1} \%$ predicted in a generalized linear model $(\mathrm{P}$ for interaction $=0.044$, and $\mathrm{P}$ 
Table 6 Association between indicators of traditional adiposities and lung function

\begin{tabular}{|c|c|c|c|}
\hline Variable & $\mathrm{FEV}_{1} \%$ predicted ${ }^{*}$ & FVC $\%$ predicted ${ }^{*}$ & $\mathrm{FEV}_{1} / \mathrm{FVC}(\%)^{\dagger}$ \\
\hline \multicolumn{4}{|l|}{ Male $(n=282)$} \\
\hline $\mathrm{BMI}, \mathrm{kg} / \mathrm{m}^{2}$ & $-0.335 \pm 0.258(0.196)$ & $-0.114 \pm 0.215(0.598)$ & $-0.121 \pm 0.113(0.286)^{\ddagger}$ \\
\hline WC, $\mathrm{cm}$ & $-0.080 \pm 0.083(0.337)$ & $-0.022 \pm 0.069(0.752)$ & $-0.024 \pm 0.037(0.511)^{\ddagger}$ \\
\hline Body fat percentage, $\%$ & $-0.276 \pm 0.103(0.008)$ & $-0.159 \pm 0.086(0.065)$ & $-0.177 \pm 0.052(0.001)^{\ddagger}$ \\
\hline \multicolumn{4}{|l|}{ Female $(n=173)$} \\
\hline $\mathrm{BMI}, \mathrm{kg} / \mathrm{m}^{2}$ & $-0.585 \pm 0.280(0.038)$ & $-0.671 \pm 0.243(0.006)$ & $0.025 \pm 0.101(0.801)^{\ddagger}$ \\
\hline $\mathrm{WC}, \mathrm{cm}$ & $-0.167 \pm 0.107(0.122)$ & $-0.213 \pm 0.093(0.024)$ & $0.004 \pm 0.040(0.913)^{\ddagger}$ \\
\hline Body fat percentage, $\%$ & $-0.153 \pm 0.164(0.352)$ & $-0.186 \pm 0.143(0.197)$ & $-0.010 \pm 0.061(0.865)^{\ddagger}$ \\
\hline
\end{tabular}

Each association between fat parameter and lung function were evaluated in a separate multiple regression analysis. Data presented as regression coefficients \pm standard error $\left(\mathrm{P}\right.$ value). *Adjusted for pack-years of smoking, HDL, and hsCRP. ${ }^{\dagger}$ Adjusted for age, height, packyears of smoking, HDL, and hsCRP. ${ }^{\ddagger} \mathrm{P}$ value for the interaction of sex to the association $\left(\mathrm{P}_{\text {interaction }}<0.05\right)$. HDL, high-density lipoprotein; hsCRP, high sensitivity C-reactive protein; BMI, body mass index; WC, waist circumference; $\mathrm{FEV}_{1}$, forced expiratory volume in 1 s; FVC, forced vital capacity.

for interaction $=0.001$, respectively).

\section{Association between traditional adiposity indicators and lung function}

Multiple regression coefficients for the relationship between traditional adiposity indicators such as BMI, waist circumference and body fat percentage, and lung function according to sex were summarized in Table 6. In males, body fat percentage demonstrated negative associations with $\mathrm{FEV}_{1} \%$ predicted and $\mathrm{FEV}_{1} / \mathrm{FVC}$ ratio $(\mathrm{P}=0.008$, and $\mathrm{P}=0.001$, respectively). In females, $\mathrm{BMI}$ and waist circumference were inversely associated with $\mathrm{FVC} \%$ predicted $(\mathrm{P}=0.006$, and $\mathrm{P}=0.024$, respectively). Among these associations, only the relationship between body fat percentage and $\mathrm{FEV}_{1} / \mathrm{FVC}$ ratio showed statistically significant interaction by the sex factor ( $\mathrm{P}$ for interaction $=0.030)$.

\section{Discussion}

In this study, the volumes of visceral fat, including pericardial fat, intrathoracic fat, and visceral abdominal fat were higher in males than in females, while subcutaneous fat in the thoracic and abdomen were higher in females than in males. In males, intrathoracic fat and visceral abdominal fat volumes were inversely related to $\mathrm{FEV}_{1} \%$ predicted, and subcutaneous thoracic fat volumes showed a negative correlation with both $\mathrm{FEV}_{1} \%$ and $\mathrm{FVC} \%$ predicted. In females, the subcutaneous thoracic fat volumes were associated with decreased both $\mathrm{FEV}_{1} \%$ and FVC\% predicted. In analyses according to fat distribution, associations that showed statistically significant interaction by sex in the interaction tests supported that the association is different between males and females.

In previous studies describing the impact of body composition on lung function, only a few found an association between thoracic fat and lung function by using directly measured volumes of fat in specific body regions such as pericardial fat and total thoracic fat $(11,13)$. Unlike previous studies, this study measured almost all of the thoracic fat volume, including intrathoracic fat, pericardial fat, and subcutaneous thoracic fat.

The Jackson Heart Study, which evaluated only pericardial fat among the thoracic fats, found that both pericardial fat and visceral abdominal fat were associated with restrictive lung patterns in both sexes. The highest quartile for pericardial fat volumes was associated with the highest odds of restrictive lung patterns [odds ratio 1.85 (95\% confidence interval, 1.22-2.79)] (11). The authors suggested that the paracrine effects of pericardial fat on the lungs and the mechanical effects of pericardial fat were associated with a restrictive lung pattern; however, the exact underlying causes remain unclear. On the contrary, this study showed that intrathoracic fat volume in males had a statistically significant association with decreased lung function, whereas pericardial fat volume exhibited no such association. One of the reasons might be the 
difference in biochemical properties between intrathoracic fat and pericardial fat. Intrathoracic fat and pericardial fat have different embryogenic origins: intrathoracic fat originates from the primitive thoracic mesenchyme; whereas pericardial fat originates from the splanchnopleuric mesoderm (23). A higher pericardial fat volume suggests an association with risk factors for cardiovascular diseases and increased severity of coronary artery disease because of its biochemical nature, increased expression of inflammatory cytokines (interleukin-6 and tumor necrosis factor-a, among others), decreased concentrations of adiponectin, and its location feature, adjacent to the coronary artery (24). Meanwhile, in some previous studies, a group of subjects with metabolic syndrome exhibited higher intrathoracic fat volumes on chest magnetic resonance imaging than a group of subjects without metabolic syndrome, with no differences in pericardial fat volume between the two groups (25). Furthermore, intrathoracic fat volumes positively correlated with visceral abdominal fat volumes (26) and were inversely associated with lung function $(27,28)$. Although the impact of increases in intrathoracic fat is less known, considering its anatomical proximity to the lung, intrathoracic fat could have a more significant effect on lung function as compared with pericardial fat. This study demonstrated that increment of both intrathoracic fat and visceral abdominal fat volumes were significantly associated with reduced lung function in males. These results suggest that the role of intrathoracic fat in the prediction of decreased lung function in males should be reconsidered. In this study, subcutaneous fat (both thoracic and abdominal) and visceral abdominal fat were inversely correlated with the $\mathrm{FEV}_{1} / \mathrm{FVC}$ ratio in males, although the reasons why have been not clarified, especially with regards to subcutaneous fat. Another study showed the inverse relationship between sagittal abdominal diameter and $\mathrm{FEV}_{1} / \mathrm{FVC}$ in males, although this relationship appeared to be less in males than in females (29).

Subcutaneous thoracic fat volumes were inversely associated with $\mathrm{FEV}_{1} \%$, and $\mathrm{FVC} \%$ predicted in both sexes. Given that subcutaneous fat envelops almost the entire thoracic cage and shows a lower expression of inflammatory genes and cytokine secretion when compared with pericardial fat volume, subcutaneous thoracic fat might be associated with decreased lung function due to mechanical effects $(24,30)$. However, a previous study reported that subcutaneous thoracic fat volume calculated on axial chest CT slices was positively associated with CRP levels and systemic adiposity markers such as BMI and fatfree masses in females (18). These previous results suggest that subcutaneous thoracic fat also has metabolic effects.

In this study, all types of visceral fat volumes were higher in males; whereas subcutaneous fat volumes were higher in females. These findings confirmed the notion that women have a higher percentage of total adipose tissue, lower visceral fat, and higher subcutaneous fat in the lower extremities than men (31). These gender-related differences in body fat distribution might induce differences in cardiometabolic risk and lung function. Although lung functions of both sexes were commonly affected by subcutaneous thoracic fat volumes through mechanical effects, lung function in males who have higher visceral fat volume might be more vulnerable to metabolic effects than in females.

It has been established that traditional adiposity indices, including BMI, waist circumference, and body fat percentage, are significantly related to lung function $(32,33)$. However, in the present study, only the association between body fat percentage and $\mathrm{FEV}_{1} / \mathrm{FVC}$ ratio differed according to sex. Neither body fat percentage nor BMI alone is reliable measurements to identify specific risk factors for the pathogenesis of diseases and can fully reflect the characteristics of body fat distribution. Compared with the traditional uses of body fat percentage, BMI or waist circumference, these direct measurements of fat volumes using CT or magnetic resonance imaging could provide more relevant information for understanding the pathophysiology of pulmonary according to sex, especially in males, and help show the correlation between lung diseases and other cardiovascular and metabolic diseases.

This study had some limitations. First, we used pulmonary function test data acquired from subjects who underwent a previously planned health-screening program. The data did not include total lung capacity, residual volume, and diffusing capacity of the lungs, as well as lung function after bronchodilator intake. The absence of these additional data made it challenging to determine lung function accurately. However, this study may represent a population without respiratory diseases that have normal or nearly normal lung function. Second, there was no consensus on a specific protocol for the measurement of subcutaneous thoracic fat. Therefore, we selected the boundary of measurements using the scapular bone as a reference point similar to those measured in another study (18). Finally, although significant effort into maintaining a standard body position for CT scanning was attempted, some variations could have influenced the accuracy of the measurements. 


\section{Conclusions}

Including all types of thoracic fat, these findings suggest that the volumes of local thoracic fat deposits were inversely associated with lung function. In addition, while subcutaneous thoracic fat volumes demonstrated the same effects in both sexes, there were sex-related differences as predictors of lung function that suggest the important roles of intrathoracic fat and visceral abdominal fat volumes in males. Future investigations involving larger cohorts and longitudinal studies are needed to evaluate the exact causeand-effect relationship between local adiposity and lung function impairment. This study may implicate further studies examining the association between local fat deposits and impaired lung function diseases such as chronic obstructive pulmonary disease, asthma, and interstitial lung disease, in which the effects of fat on lung function would be more significant.

\section{Acknowledgments}

The author thanks to Inbong Hwang from the department of radiology at the Dongguk University Ilsan Hospital for radiologic image processing and Su Jin Jeong from the Statistics Support Department at Kyung Hee Medical Science Research Institute for the statistical consulting services. This work was supported by the Dongguk University Research Fund of 2018.

\section{Footnote}

Conflicts of Interest: The authors have no conflicts of interest to declare.

Ethical Statement: The authors are accountable for all aspects of the work in ensuring that questions related to the accuracy or integrity of any part of the work are appropriately investigated and resolved. This study was approved by the Institutional Review Board of Dongguk University Ilsan Hospital (2016-146).

\section{References}

1. Frisancho AR. New standards of weight and body composition by frame size and height for assessment of nutritional status of adults and the elderly. Am J Clin Nutr 1984;40:808-19.

2. Rosenfalck AM, Almdal T, Gotfredsen A, et al. Body composition in normal subjects: relation to lipid and glucose variables. Int J Obes Relat Metab Disord 1996;20:1006-13.

3. Wolk R, Berger P, Lennon RJ, et al. Body mass index: a risk factor for unstable angina and myocardial infarction in patients with angiographically confirmed coronary artery disease. Circulation 2003;108:2206-11.

4. Goodpaster BH. Measuring body fat distribution and content in humans. Curr Opin Clin Nutr Metab Care 2002;5:481-7.

5. Fox CS, Massaro JM, Hoffmann U, et al. Abdominal visceral and subcutaneous adipose tissue compartments: association with metabolic risk factors in the Framingham Heart Study. Circulation 2007;116:39-48.

6. Lee JJ, Pedley A, Hoffmann U, et al. Association of Changes in Abdominal Fat Quantity and Quality With Incident Cardiovascular Disease Risk Factors. J Am Coll Cardiol 2016;68:1509-21.

7. Taguchi R, Takasu J, Itani Y, et al. Pericardial fat accumulation in men as a risk factor for coronary artery disease. Atherosclerosis 2001;157:203-9.

8. Jeong JW, Jeong MH, Yun KH, et al. Echocardiographic epicardial fat thickness and coronary artery disease. Circ J 2007;71:536-9.

9. Rosito GA, Massaro JM, Hoffmann U, et al. Pericardial fat, visceral abdominal fat, cardiovascular disease risk factors, and vascular calcification in a community-based sample: the Framingham Heart Study. Circulation 2008;117:605-13.

10. Akbas EM, Hamur H, Demirtas L, et al. Predictors of epicardial adipose tissue in patients with type 2 diabetes mellitus. Diabetol Metab Syndr 2014;6:55.

11. Hickson DA, Liu J, Bidulescu A, et al. Pericardial fat is associated with impaired lung function and a restrictive lung pattern in adults: the Jackson Heart Study. Chest 2011;140:1567-73.

12. Ochs-Balcom HM, Grant BJ, Muti P, et al. Pulmonary function and abdominal adiposity in the general population. Chest 2006;129:853-62.

13. Watson RA, Pride NB, Thomas EL, et al. Relation between trunk fat volume and reduction of total lung capacity in obese men. J Appl Physiol (1985) 2012;112:118-26.

14. Schweitzer L, Geisler C, Johannsen M, et al. Associations between body composition, physical capabilities and pulmonary function in healthy older adults. Eur J Clin Nutr 2017;71:389-94.

15. Obesity: preventing and managing the global epidemic. Report of a WHO consultation. World Health Organ 
Tech Rep Ser 2000;894:i-xii, 1-253.

16. Borkan GA, Gerzof SG, Robbins AH, et al. Assessment of abdominal fat content by computed tomography. Am J Clin Nutr 1982;36:172-7.

17. Mahabadi AA, Massaro JM, Rosito GA, et al. Association of pericardial fat, intrathoracic fat, and visceral abdominal fat with cardiovascular disease burden: the Framingham Heart Study. Eur Heart J 2009;30:850-6.

18. Diaz AA, Zhou L, Young TP, et al. Chest CT measures of muscle and adipose tissue in COPD: gender-based differences in content and in relationships with blood biomarkers. Acad Radiol 2014;21:1255-61.

19. Dey D, Wong ND, Tamarappoo B, et al. Computer-aided non-contrast CT-based quantification of pericardial and thoracic fat and their associations with coronary calcium and Metabolic Syndrome. Atherosclerosis 2010;209:136-41.

20. Chung SJ, Kim D, Park MJ, et al. Metabolic syndrome and visceral obesity as risk factors for reflux oesophagitis: a cross-sectional case-control study of 7078 Koreans undergoing health check-ups. Gut 2008;57:1360-5.

21. Miller MR, Hankinson J, Brusasco V, et al. Standardisation of spirometry. Eur Respir J 2005;26:319-38.

22. Choi JK, Paek D, Lee JO. Normal predictive values of spirometry in Korean population. Tuberc Respir Dis 2005;58:230-42.

23. Sacks HS, Fain JN. Human epicardial adipose tissue: a review. Am Heart J 2007;153:907-17.

24. Mazurek T, Zhang L, Zalewski A, et al. Human epicardial adipose tissue is a source of inflammatory mediators. Circulation 2003;108:2460-6.

Cite this article as: Kwack WG, Kang YS, Jeong YJ, Oh JY, Cha YK, Kim JS, Yoon YS. Association between thoracic fat measured using computed tomography and lung function in a population without respiratory diseases. J Thorac Dis 2019;11(12):5300-5309. doi: 10.21037/jtd.2019.11.54
25. Chen O, Sharma A, Ahmad I, et al. Correlation between pericardial, mediastinal, and intrathoracic fat volumes with the presence and severity of coronary artery disease, metabolic syndrome, and cardiac risk factors. Eur Heart J Cardiovasc Imaging 2015;16:37-46.

26. Sironi AM, Gastaldelli A, Mari A, et al. Visceral fat in hypertension: influence on insulin resistance and beta-cell function. Hypertension 2004;44:127-33.

27. Park YS, Kwon HT, Hwang SS, et al. Impact of visceral adiposity measured by abdominal computed tomography on pulmonary function. J Korean Med Sci 2011;26:771-7.

28. de Oliveira PD, Wehrmeister FC, Horta BL, et al. Visceral and subcutaneous abdominal adiposity and pulmonary function in 30-year-old adults: a cross-sectional analysis nested in a birth cohort. BMC Pulm Med. 2017;17:157.

29. Pekkarinen E, Vanninen E, Lansimies E, et al. Relation between body composition, abdominal obesity, and lung function. Clin Physiol Funct Imaging 2012;32:83-8.

30. Chatterjee TK, Stoll LL, Denning GM, et al. Proinflammatory phenotype of perivascular adipocytes: influence of high-fat feeding. Circ Res 2009;104:541-9.

31. Karastergiou K, Smith SR, Greenberg AS, et al. Sex differences in human adipose tissues - the biology of pear shape. Biol Sex Differ 2012;3:13.

32. Maiolo C, Mohamed EI, Carbonelli MG. Body composition and respiratory function. Acta Diabetol 2003;40 Suppl 1:S32-8.

33. Park JE, Chung JH, Lee KH, et al. The effect of body composition on pulmonary function. Tuberc Respir Dis (Seoul) 2012;72:433-40. 